\title{
Intrapulmonary metastasis of non-small cell lung cancer: A prognostic assessment
}

Takehiro Okumura, MD

Hisao Asamura, MD

Kenji Suzuki, MD

Haruhiko Kondo, MD

Ryosuke Tsuchiya, MD
From the Thoracic Surgery Division, National Cancer Center Hospital, Tokyo, Japan.

Supported in part by a Grant-in-Aid for Cancer Research (11-19) from the Ministry of Health and Welfare, Japan.

Received for publication Oct 10, 2000; revisions requested Dec 1, 2000; revisions received Dec 27, 2000; accepted for publication Jan 22, 2001.

Address for reprints: Hisao Asamura, MD, Thoracic Surgery Division, National Cancer Center Hospital, 1-1, Tsukiji 5-chome, Chuo-ku, Tokyo 104-0045, Japan (E-mail: hasamura@ncc.go.jp).

J Thorac Cardiovasc Surg 2001;122:24-8

Copyright (C) 2001 by The American Association for Thoracic Surgery

0022-5223/2001 $\$ 35.00+0 \quad \mathbf{1 2 / 1 / 1 1 4 6 3 7}$

doi:10.1067/mtc. 2001.114637
Objective: According to the revised TNM classification in 1997, intrapulmonary metastasis within the same lobe of the primary tumor is designated as T4 and intrapulmonary metastasis in a different lobe is M1. However, their prognostic implications remain unclear. To assess their prognoses, we retrospectively analyzed the postoperative survival of patients with and without intrapulmonary metastasis.

Methods: From January 1982 to December 1996, 2340 patients with non-small cell lung cancer underwent surgical resection. The survival of patients having complete resection $(\mathrm{n}=1534)$ was analyzed according to their intrapulmonary metastasis status: patients without intrapulmonary metastasis $(n=1393)$, those with metastasis in the same lobe $(n=105)$, and those with metastasis in a different lobe $(n=18)$. For comparison, patients with T4 disease without intrapulmonary metastasis in the same lobe $(n=54)$ and those with M1 disease without metastasis in a different lobe (distant $\mathrm{M} 1, \mathrm{n}=18$ ) were also analyzed.

Results: The overall 5-year survivals were as follows: no intrapulmonary metastasis, 60\%; stage T4 disease with no intrapulmonary metastasis, 34\%; pulmonary metastasis in the same lobe, $34 \%$; pulmonary metastasis in a different lobe, $11 \%$; and distant M1, 6\%. The differences in survival between patients with no pulmonary metastasis and those with metastasis in the same lobe $(P<.001, \log$-rank test) and between patients with metastasis in the same lobe and those with distant M1 $(P<.001)$ were significant. In contrast, there was no significant difference between patients with metastasis in the same lobe and those with T4 disease and no intrapulmonary metastasis or between patients with metastasis to a different lobe and those with distant M1.

Conclusions: Prognostically, intrapulmonary metastasis within the same lobe of the primary tumor was comparable with $\mathrm{T} 4$ and that in a different lobe was comparable with M1. In terms of postoperative prognosis, the revised TNM classification for intrapulmonary metastasis seems to be appropriate.

\footnotetext{
I
} $\mathrm{n}$ the TNM staging system revised in 1987 for lung cancer, ${ }^{1}$ intrapulmonary metastasis (PM) was designated as distant metastasis (M1). In contrast, in the most recent revision of the TNM staging system in $1997,2 \mathrm{PM}$ within the same lobe of the primary tumor (PMs) was designated as T4 and PM in a different lobe (PMd) as M1. The rationale for this change is based on a study by Deslauriers and associates ${ }^{3}$ that found a favorable prognosis for patients with PM compared with those with distant metastasis excluding the lung (distant M1). They stated that local spread could be a possible metastatic mechanism of PM. 

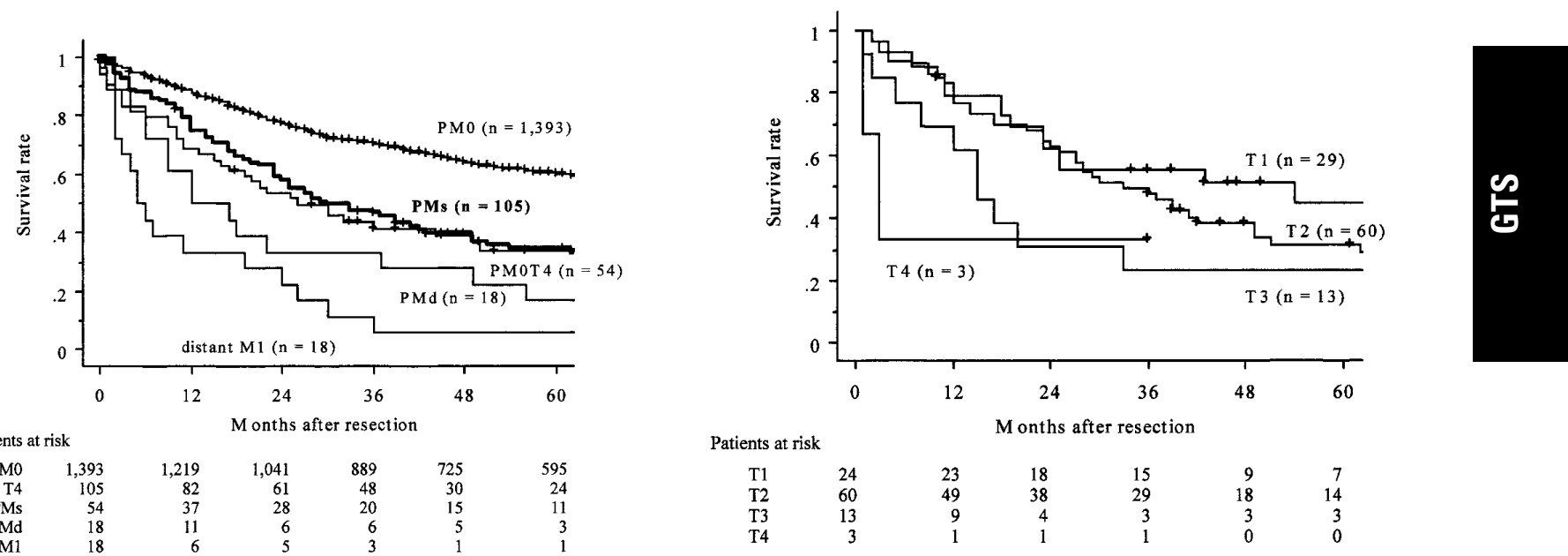
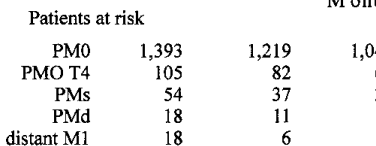

$\begin{array}{rrr}1,041 & 889 & 725 \\ 61 & 48 & 30 \\ 28 & 20 & 15 \\ 6 & 6 & 5 \\ 5 & 3 & \end{array}$

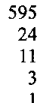

Figure 1. Overall survival curves of patients with PMO, PMOT4, PMs, PMd, and distant M1. The differences in survival between patients with PMs and with PMO and between PMs and distant M1 are significant $(P<.001$, respectively). The difference in survival between patients with PMs and with PMOT4 is not significant $(P=.823)$.

Urschel and coworkers ${ }^{4}$ claimed that the actual postoperative 5-year survival of patients with PM was $20 \%$ on the basis of a review of 11 reports, which is much higher than would be expected on the basis of clinical T4 descriptors. However, it may be questionable to conclude that the present TNM classification is inappropriate with comparison of the survival of PM patients who underwent resection and clinical T4 descriptors. No previous report has compared PMs cases with T4 cases with regard to their operative outcome, even including cases with malignant pleural effusion and dissemination in the $\mathrm{T} 4$ population. The prognostic implications of PM in lung cancer remain unclear.

We retrospectively analyzed the postoperative prognosis of patients who had non-small cell lung cancer (NSCLC) without PM (PM0), with PM, or with distant M1. Moreover, the postoperative prognosis of patients whose tumors were designated as T4 for some reason other than PM was also analyzed. To evaluate the prognostic implications of PM, we compared these prognoses.

\section{Patients and Methods \\ Patients}

From January 1982 to December 1996, 2340 patients with NSCLC underwent surgical resection at National Cancer Center Hospital, Tokyo, Japan. Of these 2340 patients, 200 (9\%) had pathologically proven PM on the basis of a postoperative pathologic study. PM was defined as a parenchymatous satellite lesion that was histologically identical to the main tumor and lacked microscopic features suggesting a primary tumor. PM was discriminated from synchronous multiple primary lung cancers on the basis of the criteria established by Martini and Melamed. ${ }^{5}$ However, cases without
Figure 2. Survival curves of patients with PMs according to the provisional pathologic T status defined by excluding PM. There was no significant difference in survival.

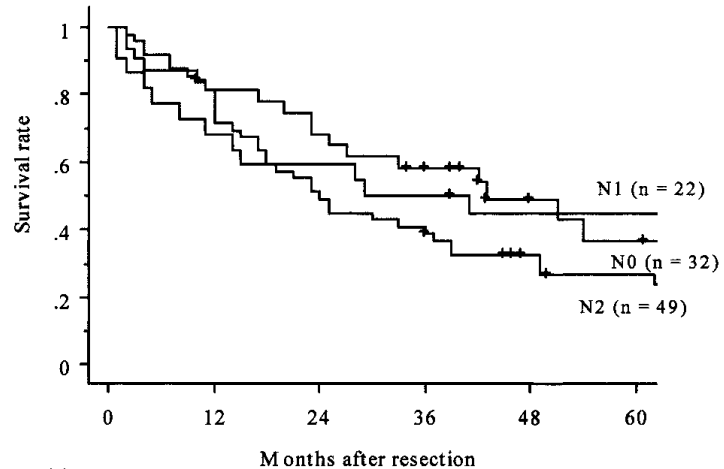

\begin{tabular}{cccccrr} 
Patients at risk & \multicolumn{5}{c}{ Months after resection } \\
N1 & 32 & 25 & 21 & 17 & 9 & 6 \\
N2 & 22 & 15 & 13 & 11 & 9 & 9 \\
N3 & 49 & 40 & 25 & 20 & 12 & 9
\end{tabular}

Figure 3. Survival curves of patients with PMs according to the pathologic nodal status. There was no significant difference in survival.

lymph node involvement were included in the PM population. Three cases in which such discrimination was difficult were excluded from this study. Of these 200 patients, 152 had PMs, and 48 had PMd. Complete resection was performed for 1393 patients with PM0, 105 with PMs, 18 with PMd, and 18 with distant M1. There were no patients with distant M1 among the PM0, PMs, and PMd populations. Complete resection was defined as segmentectomy or greater resection of the primary lesion with microscopically negative surgical margins. However, the patients designated as T4 for malignant effusion or pleural dissemination were included if the primary lesions were resected. Basically, it required mediastinal lymph node dissection but permitted a less extensive dissection if there was no macroscopically metastatic node after lymphadenectomy. 
TABLE 1. General characteristics of patients with PMO, PMs, PMd, and distant M1

\begin{tabular}{|c|c|c|c|c|}
\hline & \multicolumn{4}{|c|}{ No. of patients } \\
\hline & PMO & PMs & PMd & Distant M1 \\
\hline \multicolumn{5}{|l|}{ Age (y) } \\
\hline Mean $\pm S D$ & $62 \pm 10$ & $62 \pm 12$ & $63 \pm 14$ & $59 \pm 10$ \\
\hline Range & 26-88 & $33-82$ & $18-76$ & $34-78$ \\
\hline Sex (male/female) & $1008 / 385$ & $73 / 32$ & $12 / 6$ & $16 / 2$ \\
\hline \multicolumn{5}{|l|}{ Type of operation } \\
\hline Segmentectomy & 18 & 1 & 1 & 0 \\
\hline Lobectomy & 1194 & 82 & 10 & 14 \\
\hline Pneumonectomy & 181 & 22 & 7 & 4 \\
\hline \multicolumn{5}{|l|}{ Level of lymph node dissection } \\
\hline Less than hilar & 86 & 5 & 2 & 5 \\
\hline Hilar & 203 & 15 & 3 & 2 \\
\hline Mediastinal or more & 1104 & 85 & 13 & 11 \\
\hline \multicolumn{5}{|l|}{ Histology } \\
\hline Adenocarcinoma & 829 & 67 & 13 & 11 \\
\hline Squamous cell carcinoma & 415 & 25 & 5 & 4 \\
\hline Adenosquamous carcinoma & 27 & 5 & 0 & 2 \\
\hline Large cell carcinoma & 76 & 6 & 0 & 1 \\
\hline Carcinoid tumors & 22 & 0 & 0 & 0 \\
\hline Others & 24 & 2 & 0 & 0 \\
\hline \multicolumn{5}{|l|}{ Pathologic* } \\
\hline $\mathrm{T} 1$ & 581 & 29 & 5 & 3 \\
\hline $\mathrm{T} 2$ & 574 & 60 & 7 & 11 \\
\hline $\mathrm{T} 3$ & 184 & 13 & 6 & 3 \\
\hline T4 & 54 & 3 & 0 & 1 \\
\hline \multicolumn{5}{|l|}{ Pathologic } \\
\hline NO & 822 & 32 & 8 & 7 \\
\hline N1 & 265 & 22 & 5 & 5 \\
\hline N2 & 282 & 49 & 5 & 6 \\
\hline N3 & 24 & 2 & 0 & 0 \\
\hline Total & 1393 & 105 & 18 & 18 \\
\hline
\end{tabular}

*Provisional T status defined by excluding PM.

\section{TABLE 2. Characteristics of patients with PMd}

\begin{tabular}{lcc}
\hline & \multicolumn{2}{c}{ No. of patients } \\
\cline { 2 - 3 } Operation & $\begin{array}{c}\text { Ipsilateral } \\
\text { PMd }\end{array}$ & $\begin{array}{c}\text { Contralateral } \\
\text { PMd }\end{array}$ \\
\hline Segmentectomy + partial resection of PMd & 1 & \\
Single lobectomy + partial resection of PMd & 4 & 2 \\
Bilobectomy & 4 & \\
Pneumonectomy & 7 & \\
Total & $\mathbf{1 6}$ & $\mathbf{2}$ \\
\hline
\end{tabular}

\section{General Characteristics of the Patients}

Table 1 shows the general characteristics of each population. PMs was detected preoperatively in 10 of 105 patients, and PMd was detected preoperatively in 7 of 18 . Sixteen patients had ipsilateral PMd and 2 had contralateral PMd. Although all contralateral lesions were resected partially, 5 patients had ipsilateral PMd lesions that were resected partially, 4 had ipsilateral PMd lesions resected togeth- er with the primary tumor by bilobectomy, and 7 had such lesions resected with the primary tumor by pneumonectomy (Table 2). We defined distant M1 as distant metastasis excluding the lung that was detected before or within 1 month after complete resection of the primary lesion and required treatment for the metastatic lesion. Sites of metastasis and their treatment are shown in Table 3. Among the patients with PM0, 54 patients were designated as T4 (PM0T4). These included patients with malignant effusion or pleural dissemination if the primary lesions were resected (Table 4).

\section{Analysis and Statistics}

First, we analyzed the overall postoperative survival of patients with PM0, PM0T4, PMs, PMd, and distant M1. Second, we analyzed the survival of patients with PMs according to the provisional pathologic T status defined by excluding PM. Similarly, the survival of patients with PMs was analyzed according to the pathologic nodal status. Survival was estimated by the KaplanMeier method ${ }^{6}$ with the date of pulmonary resection as the starting date. The log-rank test was used to determine the statistical significance of differences in survival. 
TABLE 3. Characteristics of patients with distant M1

\begin{tabular}{lccccc}
\hline & \multicolumn{4}{c}{ No. of patients } \\
\cline { 2 - 6 } Treatment modality & Brain & Bone & Adrenal gland & Subcutaneous tissue & Gallbladder \\
\hline Resection & 5 & 0 & 3 & 1 & 1 \\
Radiation & 6 & 1 & 0 & 0 & 0 \\
Chemotherapy & 0 & 1 & 0 & 0 & 0 \\
Total & $\mathbf{1 1}$ & $\mathbf{2}$ & $\mathbf{3}$ & $\mathbf{1}$ \\
\hline
\end{tabular}

\section{Results}

The overall follow-up ranged from 0 to 179 months, with a median of 47 months. Complete follow-up for 5 years was obtained for 1112 patients with PM0 (45 patients with PM0T4), 90 patients with PMs, and 18 patients each with PMd and distant M1.

\section{Overall Survival}

The 5-year survivals of the patients with PM0, PM0T4, PMs, PMd, and distant M1 were 60\%, 34\%, 34\%, 11\%, and $6 \%$, respectively (Figure 1). The differences in survival between PM0 and PMs $(P<.001)$ and between PMs and distant M1 $(P<.001)$ were significant. In contrast, the survival curves for patients with PM0T4 and with PMs nearly overlapped each other, and the difference in survival between them was not statistically significant $(P=.823)$. Similarly, the difference in survival between patients with PMd and with distant M1 $(P=.113)$ was not significant, as were those between PMs and PMd $(P=.058)$ and between PM0T4 and PMd $(P=.135)$. Significant differences in survival were observed between PM0 and PM0T4, PM0 and PMd, PM0 and distant M1 $(P<.001$, respectively) and PM0T4 and distant M1 $(P=.002)$.

\section{Survival of Patients with PMs According to the Provisional Pathologic T Status}

Among the PMs population, T4 cases showed vital organ invasion but no malignant pleural effusion or pleural dissemination. The 5-year survivals of the PMs cases with T1, T2, and T3 tumors were 38\%, 32\%, and 23\%, respectively (Figure 2), whereas the 5-year survival with T4 could not be calculated because there was no patient at risk at 5 years. There was no significant difference in survival, although the T4 population was too small for a comparison (T1 vs T2, $P=.269$; T1 vs T3, $P=.116$; T1 vs T4, $P=.144 ;$ T2 vs T3, $P=.464 ;$ T2 vs T4, $P=.182$; T3 vs T4, $P=.784)$.

\section{Survival of Patients with PMs According to the Pathologic Nodal Status}

Two N3 patients were excluded from this analysis because they were too small to analyze. The 5-year survivals of the remaining PMs cases with N0, N1, and N2 nodal involve- ment were $37 \%, 40 \%$, and $24 \%$, respectively (Figure 3 ). There were no significant differences in survival (N0 vs N1, $P=.775 ; \mathrm{N} 0$ vs N2, $P=.188 ; \mathrm{N} 1$ vs N2, $P=.253)$.

\section{Discussion}

It is important to discriminate PM from synchronous multiple primary lung cancers for a discussion of the prognostic implications of PM. However, in some cases such discrimination can be difficult. Although new methods ${ }^{7,8}$ have recently been applied to this differentiation, they are not practical at present. Under these conditions it is most practical to exclude synchronous multiple primary lung cancers from PM on the basis of the criteria established by Martini and Melamed ${ }^{5}$ that have been widely accepted for a diagnosis of multiple lung cancer. Consequently, the possibility of including some cases of synchronous multiple primary lung cancer in the PM population is inevitable. However, the incidence of PM in our series was only 9\%, which is comparable with the $8 \%$ observed in the series of Deslauriers and colleagues. ${ }^{3}$ Thus, we considered that our PM population was worth analyzing.

In our series the 5-year survival of patients with PMs was $34 \%$, which was significantly worse than that of patients with PM0 and better than that of patients with distant M1. Yano, ${ }^{9}$ Okada, ${ }^{10}$ and their associates also reported that PMs cases had a worse survival than PM0 cases $(37 \%$ and $30 \%$ at 5 years, respectively). On the other hand, Shimizu, ${ }^{11}$ Fukuse, $^{12}$ and their colleagues reported that PM (PMs + PMd) cases had a better survival than distant M1 cases $(26 \%$ at 5 years). It may be safe to assume that the postoperative prognosis of patients with PMs is between those of patients with PM0 and those with distant M1. The problem is where patients with PMs should be designated in the TNM classification.

According to the latest TNM classification, ${ }^{2} \mathrm{~T} 4$ consists of 3 populations: (1) a tumor with malignant pleural, pericardial effusion, or pleural dissemination; (2) a tumor that invades the mediastinum or an adjacent vital organ such as the heart, great vessels, carina, trachea, esophagus, or vertebra; and (3) a tumor with PMs. The 5-year survival for patients in the former two T4 populations (PM0T4, $\mathrm{n}=54$; the first population underwent a resection of the primary lesion and the second population underwent a complete 
TABLE 4. Characteristics of PMO patients designated as T4

\begin{tabular}{lc}
\hline T4 descriptors & No. of patients \\
\hline Malignant pleural or pericardial effusion & 13 \\
Pleural dissemination & 14 \\
Invasion to adjacent major organ & 33 \\
Heart and great vessels & 18 \\
Vertebral body & 2 \\
Carina & 10 \\
Trachea & 2 \\
Esophagus & 1 \\
Total & $\mathbf{6 0}$ (excluding multiple conditions: 54)
\end{tabular}

resection) is $34 \%$ in our series. Macchiarini and associates ${ }^{13}$ reported that the 5-year survival of 14 patients with completely resected $\mathrm{T} 4$ tumor invading adjacent structures was $29 \%$, whereas that of 49 patients with T4 including incomplete resections was only 5\%. Similarly, Hsu and colleagues ${ }^{14}$ reported that the 5 -year survival of 25 patients with completely resected T4 tumor was $23 \%$. Although the operative indications of T4 descriptors include a selection bias, the outcome for patients with PM0T4 in our series is comparable with their results. In this study the difference in survival between patients with PMs and PM0T4 was not statistically significant. The postoperative survival of PMs cases seems to be comparable with that of $\mathrm{T} 4$ cases.

Generally, nodal status is considered to be one of the most important prognostic factors in NSCLC. ${ }^{15}$ Shimizu and coworkers ${ }^{11}$ reported the survival of PM cases according to their pathologic nodal and T status. They found that N0 cases had a better survival than $\mathrm{N} 1$ and $\mathrm{N} 2$ cases and that $\mathrm{T} 1$ cases had a better survival than T2, T3, and T4 cases. Okada and associates ${ }^{10}$ also reported a difference in survival among PM cases according to the pathologic nodal status. In contrast, no significant difference in survival was observed among the PMs cases according to either nodal or $\mathrm{T}$ status in our series. This result suggests that PM may be a strong prognostic factor equivalent to nodal status for patients with lung cancer.

PMd has been designated as M1 because it was considered to result from a spread via systemic blood circulation. The survival of PMd actually was not significantly different from that of distant organ metastasis in our study. The operative indications for patients with sublesions in a different lobe and with distant organ metastasis have a greater selection bias than those for patients with sublesions within the same lobe. The actual survivals for patients with PMd and distant organ metastasis are likely to be considerably worse than those observed in this study. Consequently, it may be futile to treat patients with PMd separately from those with distant organ metastasis. The difference in survival between patients with PMs and those with PMd was not statistically significant. Fukuse and coworkers ${ }^{12}$ reported similar results. The small number of patients with PMd in this study may have caused this result.

The present results suggest that the postoperative prognosis of patients with PMs and those with PMd are comparable with those in patients with $\mathrm{T} 4$ and with $\mathrm{M} 1$, respectively. Although our data concern comparisons of postoperative prognoses alone, they may support the revised TNM classification for lung cancer in terms of postoperative prognosis. Furthermore, PMs may be a poor prognostic factor that is equivalent to nodal status. Because there is currently no method for making a definite diagnosis of PM, the operative indications for patients with sublesions should be considered carefully.

We are grateful to the Pathology Division, National Cancer Center Research Institute, Tokyo, Japan, for their useful advice.

\section{References}

1. UICC. TNM classification of malignant tumors. 4th ed. Berlin: Springer-Verlag; 1987. p. 69-73.

2. UICC. TNM classification of malignant tumors. 5th ed. New York: Wiley-Liss; 1997. p. 93-7.

3. Deslauriers J, Brisson J, Cartier R, Fournier M, Gagnon D, Piraux M, et al. Carcinoma of the lung: evaluation of satellite nodules as a factor influencing prognosis after resection. $J$ Thorac Cardiovasc Surg. 1989;97:504-12.

4. Urschel JD, Urschel DM, Anderson TM, Antkowiak JG, Takita H. Prognostic implications of pulmonary satellite nodules: are the 1997 staging revisions appropriate? Lung Cancer. 1998;21:83-7.

5. Martini N, Melamed MR. Multiple primary lung cancers. J Thorac Cardiovasc Surg. 1975;70:606-12.

6. Kaplan EK, Meier P. Nonparametric estimation from incomplete observations. J Am Stat Assoc. 1958;53:457-81.

7. Ichinose $\mathrm{Y}$, Hara N, Ohta M. Synchronous lung cancers defined by deoxyribonucleic acid flow cytometry. J Thorac Cardiovasc Surg. 1991;102:418-24.

8. Matsuzoe D, Hideshima T, Ohshima K, Kawahara K, Shirakusa T, Kimura A. Discrimination of double primary lung cancer from intrapulmonary metastasis by p53 gene mutation. $\mathrm{Br} J$ Cancer. 1999;79:1549-52.

9. Yano $\mathrm{M}$, Arai $\mathrm{T}$, Inagaki $\mathrm{K}$, Morita $\mathrm{T}$, Nomura $\mathrm{T}$, Ito $\mathrm{H}$. Intrapulmonary satellite nodule of lung cancer as a $\mathrm{T}$ factor. Chest. 1998:114:1305-8.

10. Okada M, Tsubota N, Yoshimura M, Miyamoto Y, Nakai R. Evaluation of TMN classification for lung carcinoma with ipsilateral intrapulmonary metastasis. Ann Thorac Surg. 1999;68:326-30.

11. Shimizu N, Ando A, Date H, Teramoto S. Prognosis of undetected intrapulmonary metastases in resected lung cancer. Cancer. 1993;71:3868-72.

12. Fukuse T, Hirata T, Tanaka F, Yanagihara K, Hitomi S, Wada H. Prognosis of ipsilateral intrapulmonary metastases in resected nonsmall cell lung cancer. Eur J Cardiothorac Surg. 1997;12:218-23.

13. Macchiarini P, Silvano G, Janni A, Mussi A, Chella A, Angeletti CA. Results of treatment and lessons learned from pathologically staged T4 non-small cell lung cancer. J Surg Oncol. 1991;47:209-14.

14. Hsu CP, Hsu NY, Chen CY. Surgical experience in treating T4 lung cancer: its resectability, morbidity, mortality and prognosis. Eur J Surg Oncol. 1996;22:171-6.

15. Mountain CF, Dresler CM. Regional lymph node classification for lung cancer staging. Chest. 1997;111:1718-23. 